\title{
Renal failure, hepatitis and myocarditis in a previously healthy man
}

\author{
Seth A Climans $\mathrm{BMSC}^{1}$, Johanna M Murphy MD FRCPC ${ }^{1,2}$, \\ Gerald A Evans MD FRCPC ${ }^{1,2,3}$, M Khaled Shamseddin MD FRCPC MSc ${ }^{1,2,4}$
}

\section{CASE PRESENTATION}

A 32-year-old male migrant worker from St Lucia presented to the emergency department with a one-week history of myalgias, headache and fever. He was previously healthy. He was visiting Canada temporarily and had arrived in Canada 11 days before his presentation. One day after arriving in Canada, he ate commercially prepared hamburger patties, which he reported as "off-tasting". Five days later, he became fatigued and experienced a bilateral, intermittent headache associated with photophobia and visual aura. He noticed that his eyes were bloodshot. Three days later, he developed loose, nonwatery, nonbloody diarrhea, associated with fever and rigors, which resolved completely in two days. At presentation, he complained of lethargy, lower-extremity myalgias, tea-coloured urine and intermittent epigastric discomfort. On examination, his temperature was $37.2^{\circ} \mathrm{C}$, heart rate 87 beats/min, respiratory rate 18 breaths/min and blood pressure $113 / 72 \mathrm{mmHg}$. He had scleral icterus with conjunctival edema (Figure 1). He had no hepatosplenomegaly. His leukocyte count was $19.9 \times 10^{9} / \mathrm{L}$, hemoglobin level $124 \mathrm{~g} / \mathrm{L}$ and platelet count $24 \times 10^{9} / \mathrm{L}$. His electrolyte levels, bicarbonate levels and anion gap were within normal limits; however, his creatinine level was $352 \mu \mathrm{mol} / \mathrm{L}$ and urea level was $18 \mathrm{mmol} / \mathrm{L}$. His aspartate aminotransferase level was $310 \mathrm{U} / \mathrm{L}$, alanine transaminase level $175 \mathrm{U} / \mathrm{L}$, alkaline phosphatase level $79 \mathrm{U} / \mathrm{L}$ and total bilirubin level $191 \mu \mathrm{mol} / \mathrm{L}$. His initial creatine kinase level was $2343 \mathrm{U} / \mathrm{L}$ and troponin I level was $1.6 \mu \mathrm{g} / \mathrm{L}$. An initial electrocardiogram showed sinus rhythm with first-degree atrioventricular block and an incomplete right bundle branch block.

He was admitted with an initial, presumptive diagnosis of hemolytic uremic syndrome or thrombotic thrombocytopenic purpura, and received two units of fresh frozen plasma. A hemolysis work-up returned negative: serum lactate dehydrogenase level was $353 \mathrm{U} / \mathrm{L}$, haptoglobin level $2.9 \mathrm{~g} / \mathrm{L}$, total bilirubin level $191 \mu \mathrm{mol} / \mathrm{L}$, indirect bilirubin level $60 \mu \mathrm{mol} / \mathrm{L}$ and direct bilirubin level $131 \mu \mathrm{mol} / \mathrm{L}$. On a blood film, fragments were only rarely observed. Abdominal ultrasonography revealed hepatic periportal echogenicity, diffuse renal cortical echogenicity and no hydronephrosis. Echocardiography showed mild concentric left ventricular wall thickening and no regional wall motion abnormalities, with a preserved left ventricular ejection fraction (54\%). Furthermore, serology for cytomegalovirus, hepatitis A virus, hepatitis B virus, hepatitis $C$ virus and HIV were negative. Blood films failed to show intraerythrocytic trophozoites. Blood cultures were negative. Stool culture for Escherichia coli O157:H7 was also negative.

He remained clinically stable and had good urine output with improving kidney function. However, his leukocyte count increased to $37.6 \times 10^{9} / \mathrm{L}$ and his direct bilirubin level and erythrocyte sedimentation rate increased to $169 \mu \mathrm{mol} / \mathrm{L}$ and $110 \mathrm{~mm} / \mathrm{h}$, respectively, suggesting progression of his hepatopathy and ongoing systemic inflammation.

A diagnostic test was performed.

\section{DIAGNOSIS}

Leptospirosis serology was ordered.

The present case contains many classic feature of severe icteric leptospirosis. Jaundice, headache, conjunctival suffusion, myalgias and diarrhea are all common symptoms (1). In severe leptospirosis, a peripheral leukocytosis with left shift occurs. There tends to be a direct hyperbilirubinemia with lesser increases in transaminase levels and slight elevation of alkaline phosphatase levels, which appear to be related to the cholestasis of sepsis. Clinical and biochemical rhabdomyolysis is common (2). Electrocardiographic abnormalities are frequent, including first-degree atrioventricular block and widespread T-wave inversion (3). Leptospirosis-associated myocarditis confers a case mortality rate as high as $54 \%(1)$. Severe thrombocytopenia can occur, but this is less common and indicates a poorer prognosis (2).

After serum was obtained, the patient was started on intravenous benzylpenicillin $3 \times 10^{6}$ units every $6 \mathrm{~h}$. He quickly improved clinically. Three days later, he was discharged home on ceftriaxone $1000 \mathrm{mg}$ intravenously daily for a total of 14 days. Three weeks postdischarge, the patient was seen in the outpatient clinic and was clinically well. His creatinine level normalized to $92 \mu \mathrm{mol} / \mathrm{L}$. His leukocyte count was $8.2 \times 10^{9} / \mathrm{L}$, hemoglobin level $109 \mathrm{~g} / \mathrm{L}$ and platelet count was $441 \times 10^{9} / \mathrm{L}$. His liver enzyme levels remained mildly elevated (alanine transaminase $147 \mathrm{U} / \mathrm{L}$ and alkaline phosphatase $200 \mathrm{U} / \mathrm{L}$ ). Retrospectively, the patient reported wading in fresh water while in St Lucia two weeks before his presentation to the authors' hospital as well as seeing rodents around his house.

The serum was sent to the National Microbiology Laboratory in Winnipeg, Manitoba. Leptospirosis ELISA for immunoglobulin M was found to be positive using the Panbio Leptospira IgM ELISA (Inverness Medical Innovations Australia Pty Ltd, Australia). Interpretation criteria were as follows: $<9$ Panbio units, unreactive; 9 to 11 , equivocal; and $>11$, positive. ELISA results were confirmed using a microagglutination test beginning at a screening dilution of 1:100. Further testing revealed Leptospira interrogans serovar Autumnalis as the most likely serovar (titre 1:800).

\section{DISCUSSION}

Leptospirosis is a worldwide zoonotic infection caused by spirochetes of the genus Leptospira (2). The estimated incidence of leptospirosis ranges from 0.1 per 100,000 to 100 per 100,000 , with tropical regions having the highest incidence (4). The disease reservoir is maintained in carrier animals through persistent colonization of their proximal renal tubules. Animals, including humans, can be asymptomatic carriers. Mice, among other mammals, are important sources of transmission and excrete Leptospira in their urine (2). Human infection occurs by exposure to this urine either directly or via fresh-water contamination. Human-to-human transmission is extremely rare (1). Bacteria can infect humans through abrasions or cuts in the skin or direct ingestion of contaminated water (5). Higher-risk activities include

${ }^{1}$ School of Medicine, Queen's University; ${ }^{2}$ Department of Medicine; ${ }^{3}$ Division of Infectious Diseases; ${ }^{4}$ Division of Nephrology, Kingston General Hospital, Kingston, Ontario

Correspondence: Dr Gerald A Evans, Division of Infectious Diseases, Kingston General Hospital, Etherington Hall, Room 3013 , 76 Stuart Street, Kingston, Ontario K7L 2V7. Telephone 613-533-6619, fax 613-533-6825, e-mail evansg@queensu.ca 


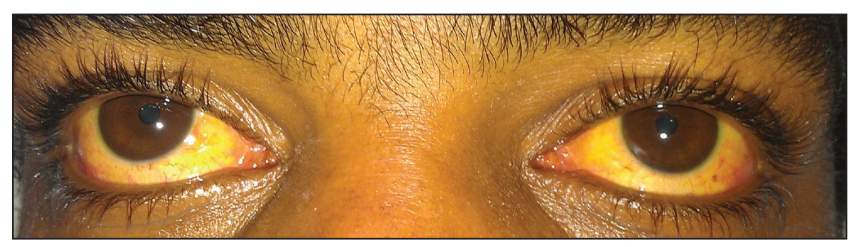

Figure 1) Photograph of the eyes of a 32-year-old man who presented with myalgias, headache and subjective fever. Scleral icterus with conjunctival edema is evident. His total bilirubin level was $256 \mu \mathrm{mol} / \mathrm{L}$ when this photograph was taken

fresh-water bathing, fishing and canoeing (6). Several cases of leptospirosis have been reported in the returning traveller $(5,6)$.

Infection can generally be divided into two categories: icteric and nonicteric leptospirosis. The icteric form is more severe and is also known as Weil's disease. This usually presents as rapidly progressive jaundice, renal failure and hemorrhagic sequelae with a variable clinical course. Intra-alveolar and interstitial pulmonary hemorrhage is most commonly observed. Diagnosis depends on a high clinical suspicion because results of serological tests generally take at least one week to return. Treatment of icteric leptospirosis remains controversial due to limited data. However, the WHO strongly recommends early treatment with antibiotics for severe leptospirosis (4). In a systematic review including 403 patients randomly assigned in four trials to receive parenteral penicillin versus placebo, penicillin reduced the length of clinical disease, but there were no differences between penicillin and placebo in all-cause mortality or length of hospitalization (7). Further trials showed no superiority of penicillin compared with cephalosporins or doxycycline (7).
Similar to antibiotic treatment of other spirochetal infections, there is a rare risk of a Jarisch-Herxheimer reaction with treatment. This reaction is caused by the host inflammatory response to endotoxins released when there is rapid widespread bacterial lysis. It typically occurs within $2 \mathrm{~h}$ of treatment and presents as fever, chills, rigors, hypotension and tachycardia (3). Most patients with icteric leptospirosis will recover without long-term sequelae.

\section{CONCLUSION}

Leptospirosis is a global zoonotic disease with potentially deadly consequences if not recognized. Acute-onset jaundice, renal failure and pulmonary hemorrhage, especially in a patient travelling from a tropical area, should raise concerns about leptospirosis, especially if initial malaria testing returns negative.

\section{REFERENCES}

1. Levett PN. Leptospirosis. Clin Microbiol Rev 2001;14:296-326.

2. Bharti AR, Nally JE, Ricaldi JN, et al. Leptospirosis: A zoonotic disease of global importance. Lancet Infect Dis 2003;12:757-71.

3. Forbes AE, Zochowski WJ, Dubrey SW, Sivaprakasam V. Leptospirosis and Weil's disease in the UK. QJM 2012;105:1151-62.

4. World Health Organization. Human leptospirosis: Guidance for diagnosis, surveillance and control. Malta: World Health Organization. < www.who.int/csr/don/en/WHO_CDS_CSR_ EPH_2002.23.pdf> (Accessed November 15, 2013).

5. Schmidhauser T, Curioni S, Bernasconi E. Acute interstitial nephritis due to Leptospira grippotyphosa in the absence of Weil's disease. Can J Infect Dis Med Microbiol 2013;24:e26-e28.

6. Lagi F, Corti G, Meli M, Pinto A, Bartoloni A. Leptospirosis acquired by tourists in Venice, Italy. J Travel Med 2012;20:128-30.

7. Brett-Major DM, Coldren R. Antibiotics for leptospirosis. Cochrane Database Syst Rev 2012;(2):CD008264. 


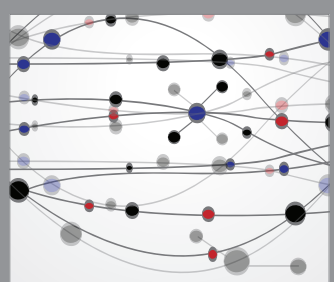

The Scientific World Journal
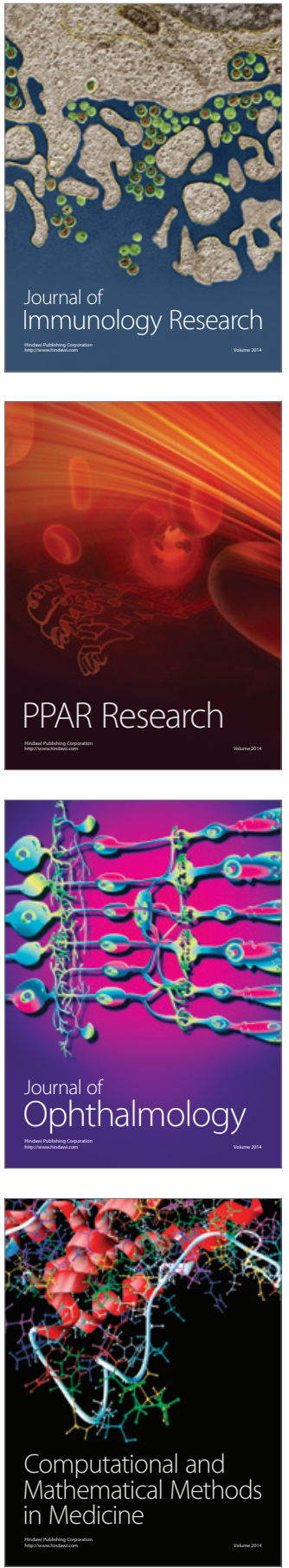

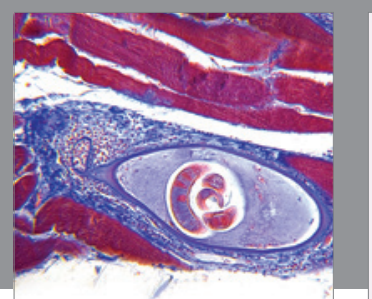

Gastroenterology Research and Practice

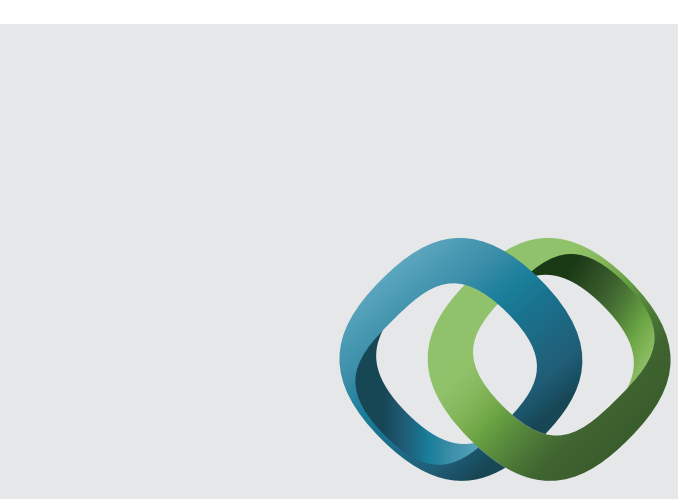

\section{Hindawi}

Submit your manuscripts at

http://www.hindawi.com
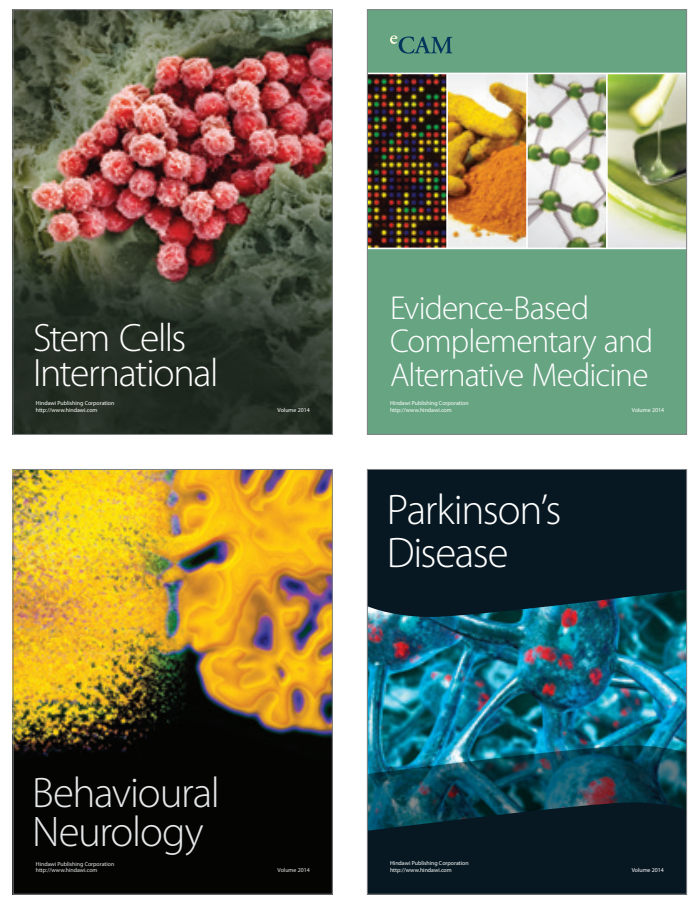
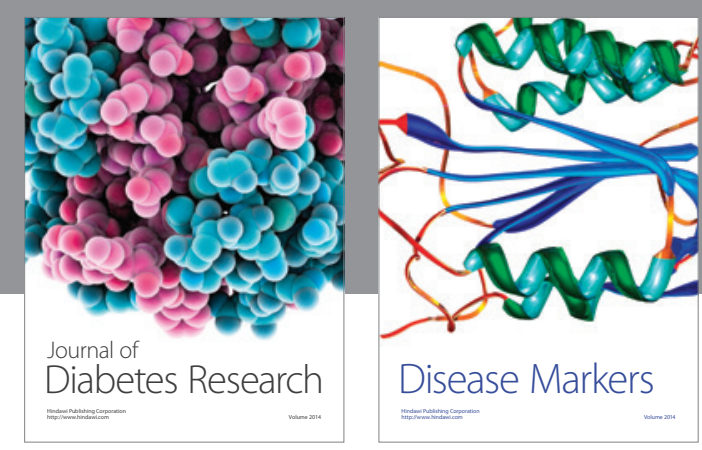

Disease Markers
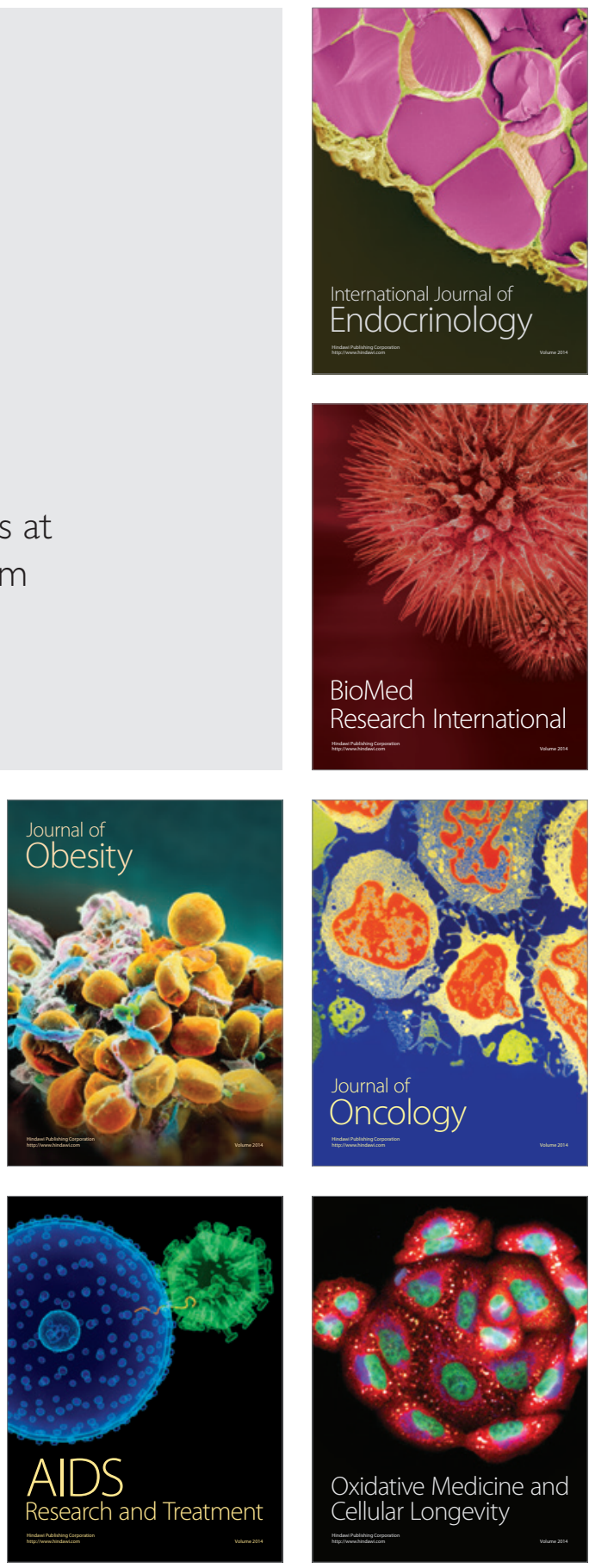